Bahadır Topuz

Murat Zor

Sercan Yilmaz

Engin Kaya

\title{
Uncommon adverse events of intravesical Bacillus Calmette-Guérin treatment: A report of three cases
}

Gulhane Training and Research Hospital, Department of Urology, Ankara, Turkey

Article history

Received: 30 December 2020; Revision Requested: 23 February 2021; Revision Received: 25 February 2020; Accepted: 21 March 2021; Published: 6 May 2021

\section{Sir,}

Intravesical instillation of Bacillus Calmette-Guérin (BCG) has been used in the treatment of high-risk superficial bladder cancer for over 40 years [1]. Despite high efficacy rates, several side effects have been reported in various conditions. Most of these adverse effects are mentioned as local side effects such as frequency, dysuria, hematuria and cystitis that all are frequent and usually resolve spontaneously [2]. Nevertheless, intravesical BCG administration may cause serious reactions such as granulomatous prostatitis, epididymo-orchitis, miliary tuberculosis, pneumonitis, septicemia and even death, approximately in $8 \%$ of patients [3]. In addition to these reactions, some very rare side effects may occur including spondylodiscitis, reactive arthritis, muscle abscess, hepatitis, vascular involvement, cutaneous involvement and ocular involvement [4]. Herein we aimed to present three extremely rare adverse events of intravesical BCG administration and discuss the treatment options according to the current literature.

The first case was a 61-year-old male patient that had been started on an induction intravesical BCG (120 mg SII ONCO BCG, India) treatment course. After the third instillation the patient admitted to our clinic with dysuria, suprapubic pain and fatigue. His medical history revealed high dose (three times higher then normal dose) intravesical BCG administration. The patient hospitalized and the BCG program was suspended. Routine blood and urinalysis were performed. Elevation of C-reactive protein (CRP) $(43.3 \mathrm{mg} / \mathrm{L})$, decrease in white blood cell count (WBC) $\left(2.6 \times 10^{3} / \mathrm{L}\right)$, and a severe decrease in platelet count $\left(2.00 \times 10^{3} / \mathrm{L}\right)$ were documented. Other routine blood and urine tests were within normal ranges. Pre-treatment platelet count was also documented to be in normal

Correspondence:

Murat ZOR, M.D.

Gulhane Research and Training Hospital, Department of Urology

06010 Etlik, Ankara, Turkey

Telephone: 00903123045607 and 00905308207615

Fax: 00903123044475

E-mail:murat804@yahoo.com ranges $\left(142.00 \times 10^{3} / \mathrm{L}\right)$. Hematological work-up revealed no other cause for this thrombocytopenic condition except high dose BCG toxicity. The patient initially treated with two units of thrombocyte replacement and the platelet count increased to $65.00 \times 10^{3} / \mathrm{L}$ on the same day. No bleeding complication was seen. No more thrombocyte replacement needed and the final platelet count was $170.00 \times 10^{3} / \mathrm{L}$ in the second week. The patient was discharged without any complication on the fifteenth day. The patient was tumor free on his two years follow-up.

To date only one case report documented bone marrow involvement secondary to intravesical BCG therapy [5]. The patient had thrombocytopenia with hypercalcemia and acute kidney disease. He also had systemic manifestations including lethargy, malaise, night sweats, weight loss and fewer. The authors succeeded to confirm M. bovis on bone marrow and successfully treated the patient with antituberculosis drugs. Our patient did not need antituberculosis medication because conservative management with platelet replacement and discontinuation of BCG was successful. Discontinuation of symptoms and improvement in laboratory findings drive us neither to perform bone marrow biopsy nor any further investigation.

The second case was a 61-year-old male patient that was started induction intravesical BCG (120 mg SII ONCO BCG, India) therapy. One month after completion of BCG therapy, he admitted to our outpatient clinic with fever, cough, weakness, penile mass, and vision loss in the left eye. The patient was hospitalized and routine blood, urine and radiological tests were performed. His physical examination was normal except round, firm, well-shaped two penile subcutaneous masses. Penile ultrasonography revealed $15 \times 5 \mathrm{~mm}$ and $10 \times 6$ $\mathrm{mm}$ anechoic cystic lesions that were located subcutaneously at the ventral side and radix of the penis (Figure-1a). In order to evaluate vision loss orbital magnetic resonance imaging (MRI) was performed. It revealed left optic disc involvement and a $3.5 \mathrm{~mm}$ mass nearby the left optic disc (Figure-1b). On 

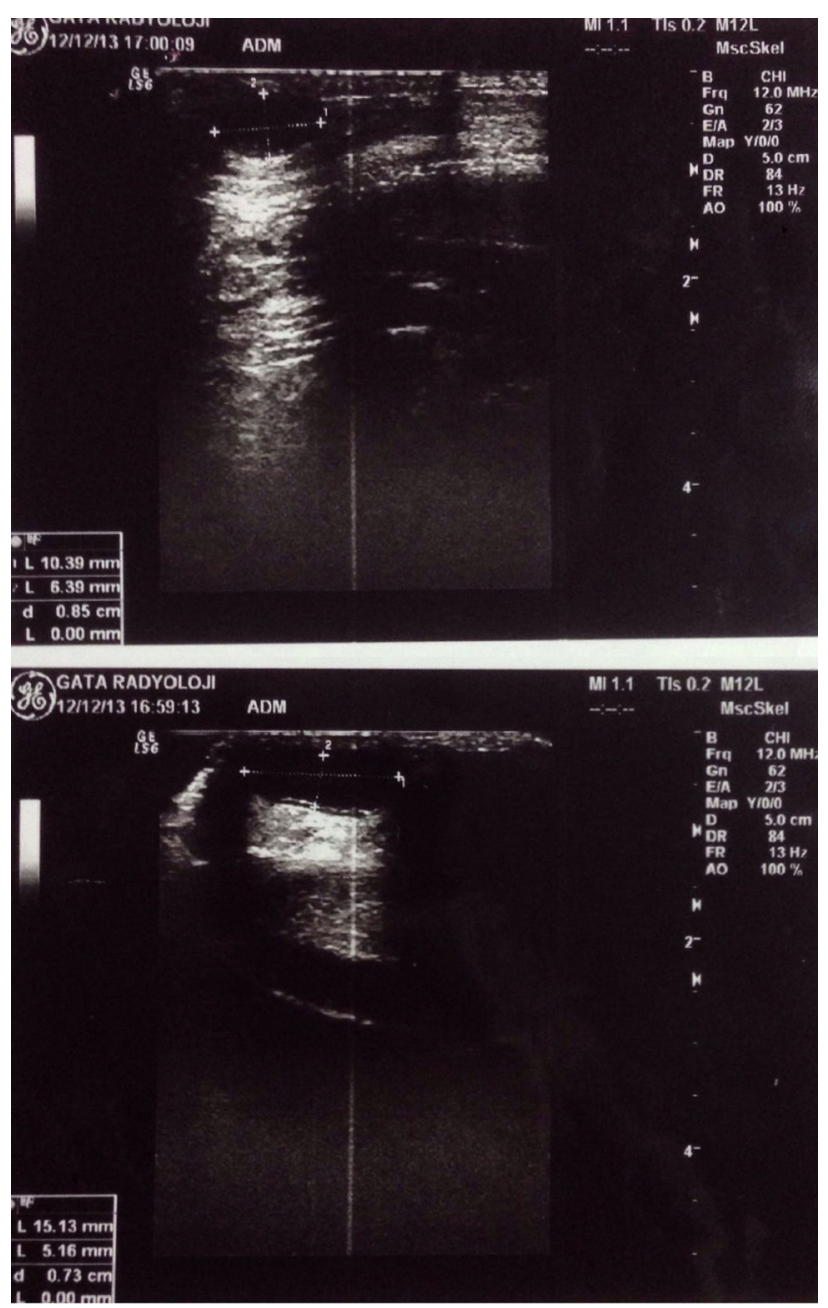

Figure $1 \mathrm{~A}$

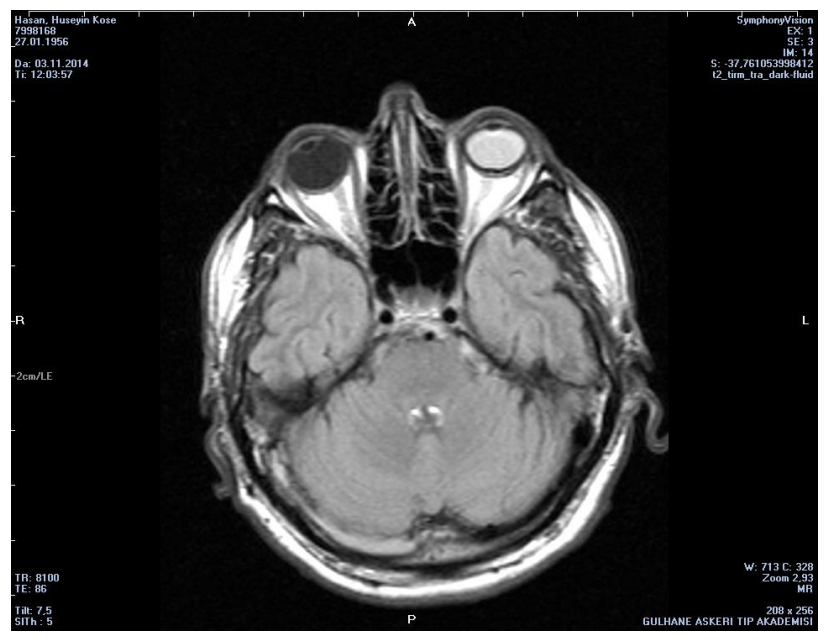

Figure 1B

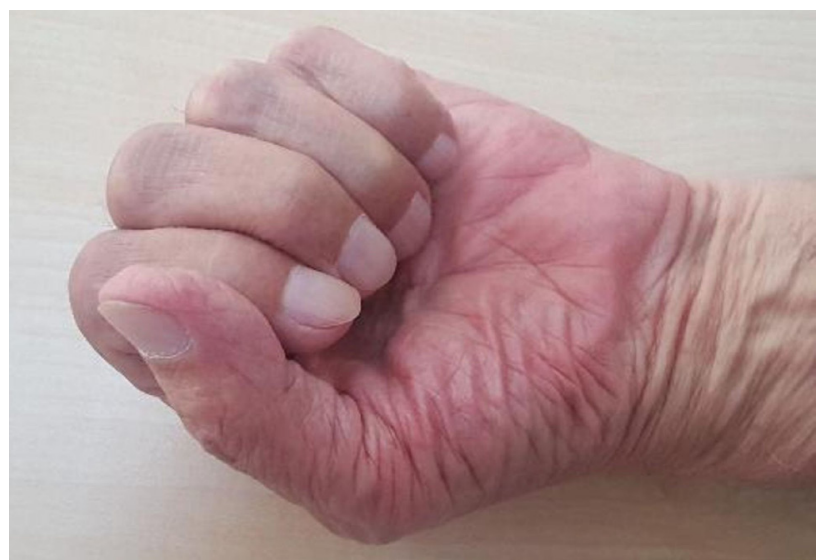

Figure 1C

Figure 1: A: Anechoic cystic lesions at the ventral side of the penis and radix of the penis. B: The left optic disc involvement. C: Swelling and erythema in his hand.

chest computed tomography, diffuse micronodules were observed in both lungs suggesting miliary tuberculosis. Cranial $M R I$ revealed multiple lesions in the left parietal lobe, the left frontal lobe, and the right temporal lobe. Simultaneously penile lesions were excised, and histopathological examination finally revealed a granulomatous inflammation. Transbronchial lung biopsy from pulmonary nodules was also confirmed the systemic granulomatous inflammation. Antituberculosis treatment was immediately initiated with isoniazid (300 mg/day), rifampicin (600 mg/day) and ethambutol (1200 mg/day). On the $1^{\text {st }}$ month follow-up, symptoms are regressed and radiological responses were seen. But his vision loss progressed and finalized with nearly $75 \%$ vision loss in the left eye. Antituberculosis treatment continued for 12 months. He had a low grade bladder tumor recurrence on $20^{\text {th }}$ month, but tumor free for the last three years.
In the literature the most frequent genitourinary complication of intravesical BCG therapy is granulomatous prostatitis, however, penile involvement is extremely rare and to our knowledge only 11 cases reported to date [6]. But, no optic disc involvement was reported to date, making our case unique in the literature. Clinical presentations of penile involvement were reported to be heterogeneous including penile edema, papules, ulcers and abscess. Our case was presented with an asymptomatic penile mass. The infrequent presentations of these complications make the treatment more difficult, thus there is no clearly defined treatment modalities. For systemic BCG complications, the European Association of Urology recommends to treat patients with 6 months course of three antituberculosis drugs [7]. Previously published cases are reported complete resolution after antituberculosis treatment [7]. Our case had two penile 
granulomas and one was excised for diagnosis. The other was completely disappeared after antituberculosis treatment confirming the current literature.

The third case was a 71-year-old patient that was receiving intravesical BCG (120 mg SII ONCO BCG, India) treatment due to bladder cancer. After 5 cycles of BCG treatment, he was admitted to our outpatient clinic with increasing pain and swelling in his hand, foot and knee joints for one month (Figure-1c). These complaints were restricting his life; moreover he was using a walking stick for these complaints. He was previously referred to orthopedics and vascular surgery outpatient clinics, and even underwent right knee arthrocentesis. Due to his medical history and physical examination, we thought that the diagnosis might be reactive arthritis secondary to BCG instillation. BCG treatment was stopped. Rheumatology consultation revealed left foot metatarsophalangeal, right knee, left hand first, second and third phalanx joint reactive arthritis. In his blood analysis WBC was $10.04 \times 10^{3} / \mathrm{ml}$, sedimentation rate was $44 \mathrm{~mm} / \mathrm{h}$, ASO was $405 \mathrm{IU} / \mathrm{ml}$, CRP was $14.9 \mathrm{~g} / \mathrm{dl}$, and rheumatoid factor (RF) was $<20 \mathrm{IU} / \mathrm{ml}$. Basic blood immunologic autoantibodies, serological and virological test results were all normal. The patient was started methylprednisolone $16 \mathrm{mg}$ twice a day, naproxen twice a day and calcium carbonate + vitamin D3 once a day. He had a significant recovery on the first week. The steroid treatment was continued for six months. The patient was well and tumor free on two-year follow-up.

Arthritis is a rare complication of BCG treatment. Reported prevalence is variable, ranging between $0.5 \%$ and $3.6 \%$ [8]. Polyarthritis is the most common clinical presentation that can be symmetric or asymmetric with similar prevalence. Mono-oligoarthritis is less common seen and tend to be in asymmetric pattern [8]. Knee and ankle are the most effected joints. It has a favorable course and vast majority of cases can be successfully treated with NSAID monotherapy, corticosteroid monotherapy or in combination. It is reported that joint symptoms last about within two months and fully recover (93.2\%) within six months [8]. Consistently with the literature our case was presented with oligo-arthritis involving knee, ankle and small hand joints, and he was successfully treated with NSAID and steroid combination.

Intravesical use of BCG has played a major role in the treatment of non-muscle invasive bladder carcinoma since its advent in 1976. The mechanism of action is poorly understood and highly controversial; however, it is generally thought to be secondary to a kind of nonspecific local immune system stimulation [2]. However playing a central role in bladder cancer treatment, intravesical BCG may manifest with a variety of complications ranging from cystitis and death. The most important and key step of the management of BCG complications is the prompt recognition of the clinical scenario However, wide variety of these conditions make diagnosis sometimes challenging for specialists. The mechanism underlying the complications is also not fully understood [9]. While some authors advocate hypersensitivity reactions secondary to BCG therapy, some others suggest an infectious disease due to dissemination of M. bovis [10]. Local complications of intravesical BCG are generally mild and can be easily treated with nonsteroidal anti-inflammatory drugs (NSAID), antipyretics, antispasmodics and anticholinergic drugs. But antituberculosis therapy may be needed for systemic complications with more severe symptoms. Rare systemic and loco-regional complications are not frequent and unfortunately due to its rarity diagnosis is sometimes challenging.

\section{FUNDING}

None to declare.

\section{CONFLICTS OF INTEREST}

The authors declare that they have no conflict of interest.

\section{REFERENCES}

1. Morales A, Eidinger D, Bruce AW. Intracavitary Bacillus Calmette-Guerin in the treatment of superficial bladder tumors. J Urol. 1976;116(2):180-183. doi: 10.1016/S0022-5347(17)58737-6.

2. Green DB, Kawashima A, Menias CO, Tanaka T, Redelman-Sidi G, Bhalla S, Shah R, King BF. Complications of Intravesical BCG Immunotherapy for Bladder Cancer. Radiographics. 2019 JanFeb;39(1):80-94. doi: 10.1148/rg.2019180014.

3. Liu Y, Lu J, Huang Y, Ma L. Clinical Spectrum of Complications Induced by Intravesical Immunotherapy of Bacillus Calmette-Guérin for Bladder Cancer. J Oncol. 2019;2019:6230409. doi: 10.1155/2019/6230409.

4. Pérez-Jacoiste Asín MA, Fernández-Ruiz $M$, López-Medrano $F$,

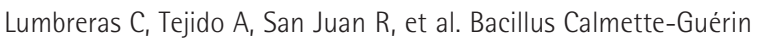
(BCG) infection following intravesical BCG administration as adjunctive therapy for bladder cancer: incidence, risk factors, and outcome in a single-institution series and review of the literature. Medicine (Baltimore). 2014; 93(17):236-254. doi: 10.1097/ MD.0000000000000119.

5. Nayar N, Briscoe K. Systemic Bacillus Calmette-Guerin sepsis manifesting as hypercalcaemia and thrombocytopenia as a complication of intravesical Bacillus Calmette-Guerin therapy. Intern Med J. 2015; 45(10):1091-2. doi: 10.1111/imj.12876.

6. Lestre SI, Gameiro CD, João A, Lopes MJ. Granulomas of the penis: a rare complication of intravesical therapy with Bacillus Calmette-Guerin. An Bras Dermatol. 2011; 86(4):759-62. doi: 10.1590/ s0365-05962011000400021.

7. Witjes JA, Palou J, Soloway M, Lamm D, Brausi M, Spermon JR, et al. Clinical practice recommendations for the prevention and management of intravesical therapy-associated adverse events. Eur Urol Suppl. 2008;7:667-74. Doi: 10.1016/j.eursup.2008.08.001.

8. Freixa M, Úria S, da Silva GN. Reactive Arthritis - A Rare Complication of Intravesical BCG Instillation. Eur J Case Rep Intern Med. 2020;7(3):001448. doi: 10.12890/2020_001448. 
9. Waked R, Choucair J, Chehata N, Haddad E, Saliba G. Intravesical Bacillus Calmette-Guerin (BCG) treatment's severe complications: A single institution review of incidence, presentation and treatment outcome. J Clin Tuberc Other Mycobact Dis. 2020 Feb 3;19:100149. doi: 10.1016/j.jctube.2020.100149.

10. Gonzalez OY, Musher DM, Brar I, Furgeson S, Boktour MR, Septimus EJ, et al. Spectrum of bacille Calmette-Guérin (BCG) infection after intravesical BCG immunotherapy. Clin Infect Dis. 2003; 36(2):1408. doi: $10.1086 / 344908$. 